\title{
Local immune and microbiological responses to mucosal administration of a Liposome-TLR agonist immunotherapeutic in dogs
}

William Wheat ${ }^{*}$, Lyndah Chow, Alana Kuzmik, Sirikul Soontararak, Jade Kurihara, Michael Lappin and Steven Dow

\begin{abstract}
Background: Non-specific immunotherapeutics have been evaluated previously in dogs, primarily for cancer treatment. However, there remains a need for a more broadly targeted, general purpose immunotherapeutic capable of activating innate immune defenses for non-specific protection or early treatment of viral and bacterial infections. To address need, our group has developed a liposomal immune stimulant (liposome-TLR complexes, LTC) containing TLR 3 and 9 agonists specifically designed to activate mucosal immune defenses in sites such as nasal cavity and oropharynx, following topical delivery. In this study, we evaluated the local immune stimulatory properties of LTC in vitro and in healthy purpose-bred dogs, including activation of cellular recruitment and cytokine production. The ability of LTC treatment to elicit effective antiviral immunity was assessed in dogs following a canine herpesvirus outbreak, and the impact of LTC treatment on the local microbiome of the oropharynx was also investigated.
\end{abstract}

Results: These studies revealed that LTC potently activated innate immune responses in vitro and triggered significant recruitment of inflammatory monocytes and T cells into the nasal cavity and oropharynx of healthy dogs. Administration of LTC to dogs shortly after an outbreak of canine herpesvirus infection resulted in significant reduction in clinical signs of infection. Interestingly, administration of LTC to healthy dogs did not disrupt the microbiome in the oropharynx, suggesting resiliency of the microflora to transient immune activation.

Conclusions: Taken together, these results indicate that LTC administration mucosally to dogs can trigger local innate immune activation and activation of antiviral immunity, without significantly disrupting the composition of the local microbiome. Thus, the LTC immune stimulant has potential for use as a non-specific immunotherapy for prevention or early treatment of viral and bacterial infections in dogs.

Keywords: Cytokine, T cells, Monocytes, Microbiome, Immunity, Immunotherapy, Infectious disease, Canine, Herpesvirus, Antimicrobial resistance, Liposomes, Toll-like receptor agonists

\section{Background}

Dogs are susceptible to infection with a multitude of different viral and bacterial pathogens that cause respiratory tract infection and illness, including viral agents such as influenza, parainfluenza, and herpesviruses and bacterial pathogens such as Bordetella and mycoplasmas

\footnotetext{
* Correspondence: william.wheat@colostate.edu

From the Center for Immune and Regenerative Medicine and the Center for Companion Animal Studies, Department of Clinical Sciences, College of Veterinary Medicine and Biomedical Sciences, Colorado State University, 80523, Ft. Collins, Colorado, CO, USA
}

[1-3]. Though vaccines are available to prevent some of these infections, in cases where animals are crowded or stressed (e.g., boarding or day care facilities or airline flights) it may not be possible to vaccinate in time to prevent infection, or vaccine immunity may decline due to stress-induced immune suppression. With some pathogens, it is difficult to induce effective or durable immunity (e.g., Bordetella canis), and some pathogens may rapidly evolve to escape specific immunity (e.g., canine influenza [4]). For these reasons, there is currently a 
need for an immunotherapeutic capable of rapidly generating non-specific immune activation and protection from a diverse array of potential canine pathogens.

A number of immunotherapeutics have been developed and evaluated as potential cancer immunotherapeutics in dogs. For example, live Mycobacterium bovis and cell wall extracts from yeast and bacteria have all been evaluated for anti-tumor activity in dogs, typically following direct intra-tumor administration [5, 6]. Perhaps the best studied tumor immunotherapeutic has been the NOD like receptor agonist muramyl tripeptide (MTP), which has demonstrated impressive anti-tumor activity in multiple dog models [7-12]. Mechanistically, MTP immunotherapy was shown to activate macrophage activity and TNF $\alpha$ production in the lungs of treated animals [13-15]. Our laboratory has previously evaluated the use of liposome-TLR complexes (LTC) which potently activate type I innate immune responses, for immunological activity in dogs with several types of cancer, including metastatic osteosarcoma $[16,17]$. Unlike the case with cancer immunotherapy, there are few non-specific immune stimulants with demonstrated activity against viral or bacterial pathogens in dogs.

We previously demonstrated in rodent infection models that cationic liposome-TLR complexes (LTC) containing non-coding plasmid DNA as a TLR9 agonist could potently activate innate immune responses and elicit highly effective protection against a variety of lethal viral and bacterial infections following mucosal administration of LTC via the intranasal route [18-23]. Moreover, we recently reported that LTC administered intranasally to cats could generate effective local immune activation and protection against FHV-1 [22, 24]. Therefore, we hypothesized that LTC could also generate effective prophylactic or early therapeutic immunity in dogs following mucosal administration. To address this question and evaluate feasibility of the new approach to infectious disease immunotherapy, we modified the original LTC to more specifically target mucosal immunity and to broaden the scope of innate immune activation, to include both TLR3 and TLR9 agonists.

In the present report, modified LTC [24] were evaluated for activation of innate immune responses in dogs, using both in vitro and in vivo assays. The studies focused on induction of local immune activation in the nasal cavity and oropharynx of dogs following intranasal administration of LTC to healthy Beagle dogs, and on whether such local immune activation could generate non-specific protection from viral infection. Finally, the impact of LTC administration on the microbiome of the oropharynx of dogs was investigated. Taken together, these studies provided convincing evidence that LTC potently activate local mucosal innate responses in the upper airways of dogs, accompanied by induction of non-specific anti-viral protective immunity.

\section{Results \\ LTC administration triggers cellular activation of dog leukocytes in vitro}

To determine whether LTC treatment stimulated immune cell activation of canine leukocytes in vitro, PBMC were purified from whole blood and plated at $1 \times 10^{6}$ cells/well and treated with several different concentrations of LTC to determine whether production of two key innate cytokines (IFNY and TNF $\alpha$ ) was upregulated. Supernatants were collected from PBMC cultures following $24 \mathrm{~h}$ stimulation with LTC, and IFN $\gamma$ and TNF $\alpha$ concentrations were assessed by ELISA (Fig. 1). These studies revealed that LTC treatment stimulated significant dose-dependent, increased secretion of IFN $\gamma$ and TNF $\alpha$. It should also be noted that LTC doses $\geq 10 \mu \mathrm{l} / \mathrm{ml}$ decreased cytokine production, due to induction of cytotoxicity, which has been reported previously with liposomeTLR therapeutics [25].

\section{Macrophage treatment with LTC triggers TNFa production and upregulated expression of $\mathrm{MHCII}$}

To assess the response of macrophages to LTC, monocyte-derived macrophage (MDM) cultures were treated with $0.5,1.0,5.0$ and $10 \mu \mathrm{l} / \mathrm{ml}$ of LTC for $24 \mathrm{~h}$ and supernatants were collected for TNF $\alpha$ analysis by ELISA. In addition, cells were detached and immunostained for flow cytometric assessment of MHCII expression. In Fig. 2, treatment of MDM with increasing dosages of LTC was found to stimulate release of increasing amounts of TNFo (Fig. 2a). In addition, LTC treatment stimulated up-regulation of surface MHCII expression by MDM (Fig. 2b). Higher concentrations of LTC ( $\geq 10 \mu \mathrm{l} / \mathrm{ml})$ led to cytoxicity and decreased release of TNF $\alpha$.

\section{LTC activate macrophage bactericidal activity}

We next evaluated whether macrophage activation by LTC might also be accompanied by induction of bactericidal activity, since activated monocytes and macrophages are likely to interact with bacterial pathogens in the upper respiratory tract. Monocyte-derived macrophages from 3 dogs were treated with LTC for $24 \mathrm{~h}$ prior to inoculation with a methicillin-resistant clinical isolate of Staphylococcus pseudointermedius, as noted in Methods. Macrophage killing of $S$. pseudointermedius by control and LTC-activated macrophages was evaluated $3 \mathrm{~h}$ after infection (Fig. 3). These studies revealed that LTC treatment triggered significant killing of $S$. pseudointermedius by macrophages, indicating that macrophage activation and upregulated MHCII expression was also accompanied by increased bactericidal activity. The 


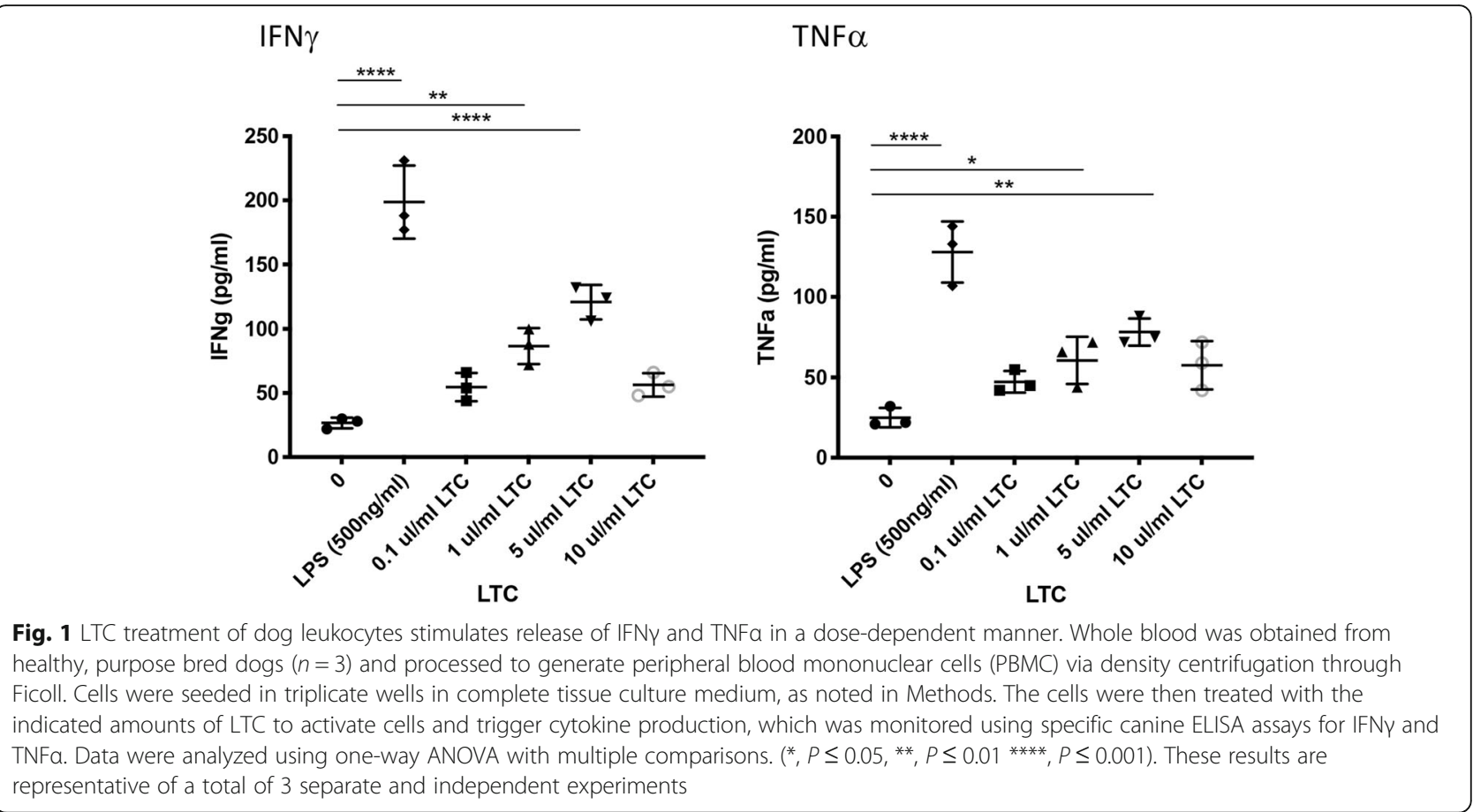

macrophage killing activity induced by LTC treatment was comparable to that elicited by IFN $\gamma$ treatment of macrophages (data not shown).

\section{LTC adherence and uptake by canine epithelial cells}

Studies were done next to assess adherence, uptake and internalization of LTC by dog epithelial cells, since activation of TLR3 and TLR9 only occurs in the intracellular endosomal compartment of cells [16]. To address this question with respect to the assessment of epithelial cells most likely to take up LTC complexes initially in the nose and oropharynx, LTC were fluorescently tagged and incubated with a canine squamous cell carcinoma cell line (CSCAG891) established previously in our laboratory (Fig. 4). Nearly confluent cultures of CSCAG891 cells seeded in 6-well plates were incubated
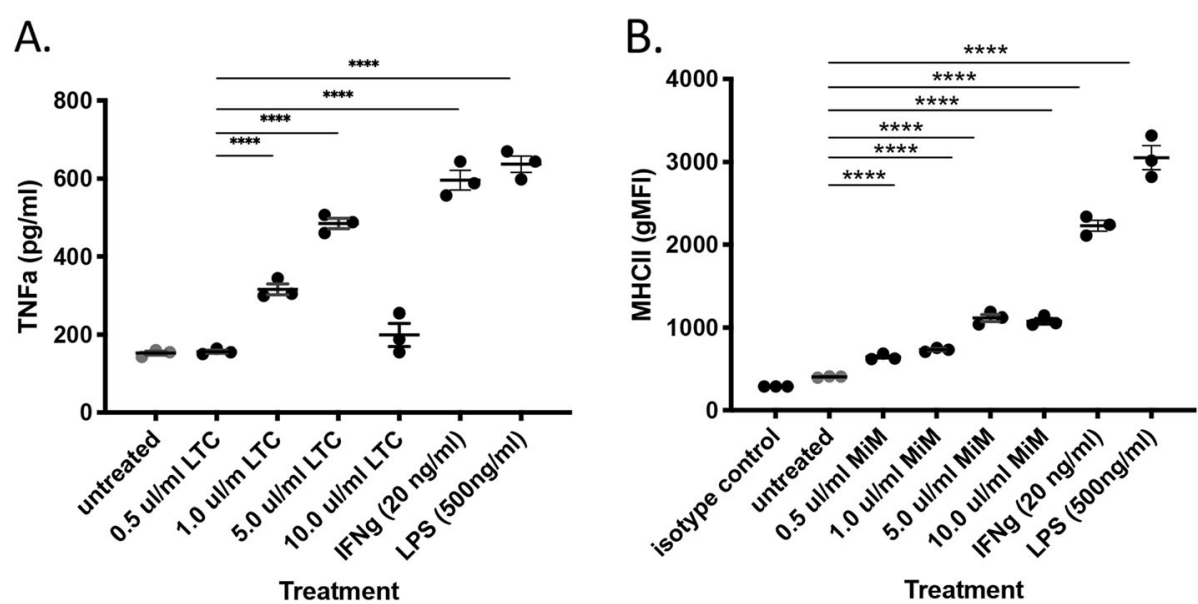

Treatment

Fig. 2 LTC treatment of dog macrophages stimulates TNFa production and upregulation of MHCIl expression. Whole blood was obtained from 3 dogs and processed to generate PBMCs, as noted in Fig. 1. Monocytes were enriched by plastic adherence of PBMC to triplicate wells of a 24well plates and differentiated to macrophages by incubation in M-CSF, as noted in Methods. Macrophages were ether untreated or treated for $18 \mathrm{~h}$ with either $1 \mathrm{ul} / \mathrm{ml}, 5 \mathrm{ul} / \mathrm{ml}, 10 \mathrm{ul} / \mathrm{ml}$ of LTC or $10 \mathrm{ng} / \mathrm{ml}$ canine IFNg or $500 \mathrm{ng} / \mathrm{ml} \mathrm{LPS}$. Secretion of TNFa in supernatants was assessed by canine TNFa ELISA (panel a). Expression of MHCIl was assessed by flow cytometry (panel b), as noted in Methods. Data were analyzed using oneway ANOVA with multiple means comparisons. ( $\left.{ }^{*}, P \leq 0.05,{ }^{* *}, P \leq 0.01, P \leq 0.001\right)$. These results are representative of a total of 3 separate and independent experiments 


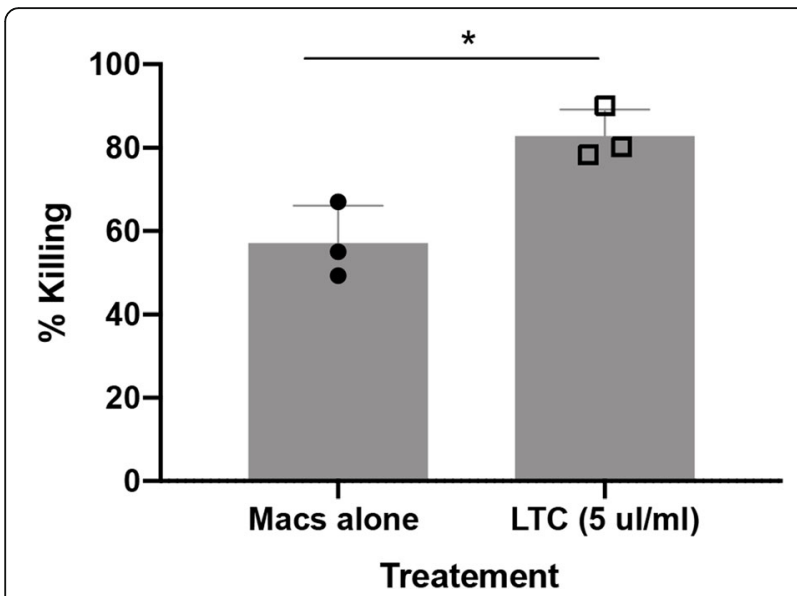

Fig. 3 Macrophages activated by LTC exhibit increased bactericidal activity. Monocyte-derived macrophages (MDM) from 3 dogs were treated with LTC for $24 \mathrm{~h}$, after which the cells were infected with a canine methicillin-resistant strain of S. pseudointermedius at an $\mathrm{MOI}$ of 5 and killing of internalized bacteria was assessed by comparing CFU from macrophage cultures in untreated vs. LTC treated cells. Comparisons between groups were done using ANOVA, followed by Tukey multiple means post-test. Analyses were performed using Prism 8 software (GraphPad, La Jolla, CA). For all analyses, statistical significance was determined if $p \leq 0.05$

with $10 \mu \mathrm{l}$ LTC labeled with green fluorescent Topfluor-labeled cholesterol (see Methods) per well for various amounts of time and cellular uptake was assessed by confocal microscopy (Fig. 4a) and flow cytometry (Fig. 4b).

We found that incubation with LTC resulted in significant uptake and intracellular distribution of the LTC complexes (Fig. 4). In addition, flow cytometry revealed rapid uptake of LTC at various time points following in vitro incubation with CSCAG891 cells. The geometric mean fluorescence intensity (gMFI) was measured by flow cytometry and plotted in Fig. 4b. These results establish that LTC are readily taken up and internalized by dog epithelial cells, which predicts that LTC would also undergo uptake by the oropharyngeal and nasal epithelium in vivo.

\section{Intranasal and oral mucosal administration of LTC results} in immune cell infiltration and activation of upper airway and oropharyngeal tissues in dogs

Healthy adult dogs ( $n=6$ per group) were treated with a single administration of LTC, delivered by both the intranasal and oral routes, as described in Methods. Cellular responses to LTC administration were evaluated over time in treated animals. Nasal lavage and oropharyngeal swab samples were obtained prior to treatment, and at $24 \mathrm{~h}, 72 \mathrm{~h}$ and 7 days after treatment. Cells from the nasal cavity and oropharynx were obtained by nasal lavage and swabbing the oropharynx, respectively (Fig. 5). Cells obtained from the two sites were analyzed by flow cytometry. T lymphocytes are identified as $\mathrm{CD}^{+}$cells, B cells were identified as $\mathrm{CD} 21^{+}$cells, monocytes were identified as $\mathrm{CD} 11 \mathrm{~b}^{+}$and $\mathrm{CD} 14^{+}$positive cells, and neutrophils were identified based on typical forward versus side-scatter characteristics.

The percentages of each cell type in nasal lavage samples over time were plotted as pie chart diagrams, as noted in Fig. 5. For nasal lavage samples, compared to pre-treatment samples, samples obtained following LTC treatment had significant increases in the percentage of $\mathrm{CD}^{+} \mathrm{T}$ cells at $72 \mathrm{~h}$ and 7 days post treatment. The percentage of monocytes was increased significantly in nasal lavage fluid, from $8 \%$ of pre-treatment lavage cells to $34 \%$ of lavage cells at $72 \mathrm{~h}$ and remained elevated at $22 \%$ after 7 days. The percentage of B cells significantly increased (from 2 to $6 \%$ ) in nasal lavage samples at $24 \mathrm{~h}$ after LTC treatment and further increasing to $13 \%$ at 72 $\mathrm{h}$ and remained elevated at $6 \%$ at 7 days post treatment. Neutrophils in nasal lavage fluid initially rose from 2 to $8 \%$ at $24 \mathrm{~h}$ after LTC treatment but declined to pretreatment levels in $72 \mathrm{~h}$ remaining at pre-treatment percentages at 7 days.

Cell samples obtained by swabs from the oropharynx (OP) had modest but significant increases in T cells following LTC treatment, increasing from $1 \%$ at pre-treatment levels to $4 \% 24 \mathrm{~h}$ post-treatment and remaining between 3 and $4 \%$ of oropharyngeal cells at $72 \mathrm{~h}$ and 7 days post-treatment (Fig. 5). The percentages of monocytes increased from 25 to $36 \%$ at $24 \mathrm{~h}$ and then returned to near or below pre-treatment values at $72 \mathrm{~h}$ and 7 days following LTC administration. The average percentage of B cells modestly increased in the oropharynx $24 \mathrm{~h}$ after LTC treatment from 1 to $3.5 \%$, remained near those levels at $72 \mathrm{~h}$ post treatment and decreased to $2 \%$ by 7 days. Neutrophils increased from 2 to $16 \%$ after $24 \mathrm{~h}$ and declined to $13 \%$ in $72 \mathrm{~h}$ and returned to pre-treatment levels 7 days after LTC administration.

\section{Monocytes from both nasal lavage and oropharyngeal samples exhibit increased expression of MHCII 1 week after LTC treatment}

Up-regulation of MHCII expression is a key phenotypic feature of activated monocytes [26] . Therefore, MHCII expression by monocytes obtained pre-treatment, and $24 \mathrm{~h}, 72 \mathrm{~h}$ and 7 days after LTC administration was determined by flow cytometric analysis. This analysis revealed that MHCII expression was significantly upregulated on monocytes isolated from both nasal lavage fluid (Fig. 6a) and oropharyngeal swabs (Fig. 6b) 7 days after LTC treatment, indicative of immune activation following LTC administration. The upregulation of MHCII expression by monocytes following in vitro LTC treatment was also observed (see Fig. 2). These data indicate therefore that LTC treatment upregulated MHCII 

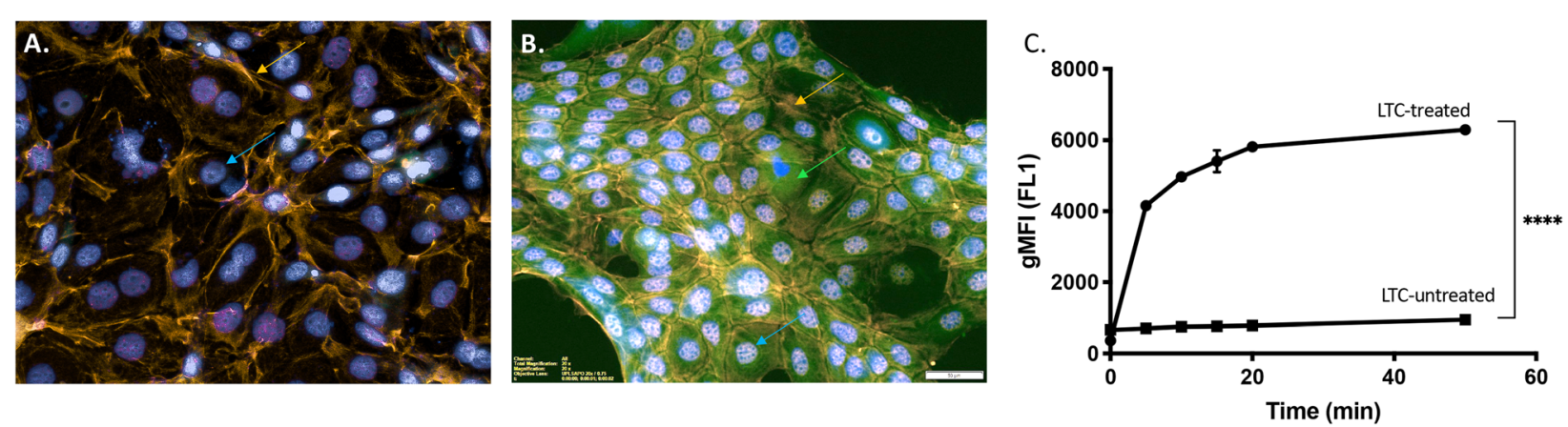

Fig. 4 The canine squamous cell carcinoma epithelial cell line CSCAG891 binds rapidly to LTC. A canine squamous cell carcinoma cell line, CSCA G891, was established from an excised canine carcinoma tumor. Cells were grown in 6-well plates to approximately $90 \%$ confluence in DMEM 10\% FBS. Triplicate cultures of CSCAG891 cells were treated with either nothing (a) or $10 \mu$ l of TopFluor ${ }^{\circledast}$-labeled LTCs for 5 min followed by washing 3X in medium (b). For microscopy, images of CSCAG891 cells either untreated or treated with $10 \mu \mathrm{l}$ of TopFluor-labeled (green arrow) CSCAG cells were subsequently stained with the cross-reactive human epithelial cell marker EpCAM (orange arrows) and nuclei stained with DAPI (blue arrows). For analysis of adherence of LTC to CSAG891 cells, cells were treated with LTC and removed from wells by treatment with trypsin, filtered and assayed by flow cytometric analysis for TopFluor ${ }^{\oplus}$ - stained cells over a period of 50 min, (c). Measured green channel (FL1) gMFI for triplicate samples was compared to unstained controls over each time point and differences were analyzed by ANOVA with a Geisser-Greenhouse's epsilon value of 0.2802 . $(* * * *, P \leq 0.001)$

expression by monocytes, most likely by inducing production of IFNY and TNFa.

To assess the impact of LTC treatment on expression of other inflammatory cytokines, RNA was extracted from cells obtained by nasal swab or oropharyngeal swabs obtained pre-treatment and on day 1 , day 3 , and day7, and subjected to $q$ RT-PCR to assess expression of IFN $\gamma$, IL-8, IL-12p40, and MCP-1 (Fig. 7). These studies revealed that LTC treatment triggered significant upregulation of expression of mRNA for two key innate immune cytokines (IFNY and IL-8) along with numerical increases in expression of mRNA encoding IL-12 and MCP - 1. These results are consistent with broad, local induction of key anti-viral and anti-bacterial cytokines in upper airway tissues following topical administration of LTC in healthy dogs.

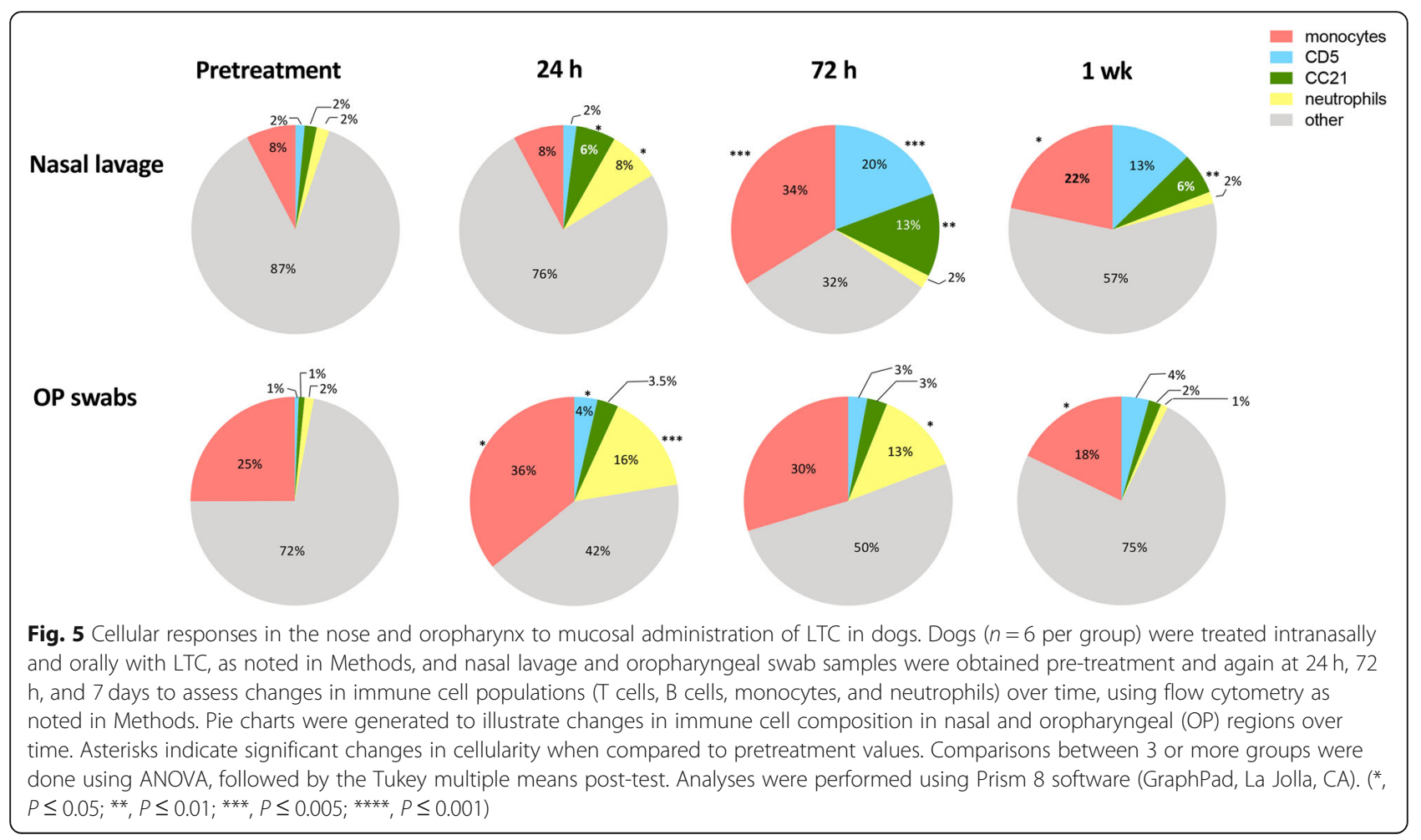



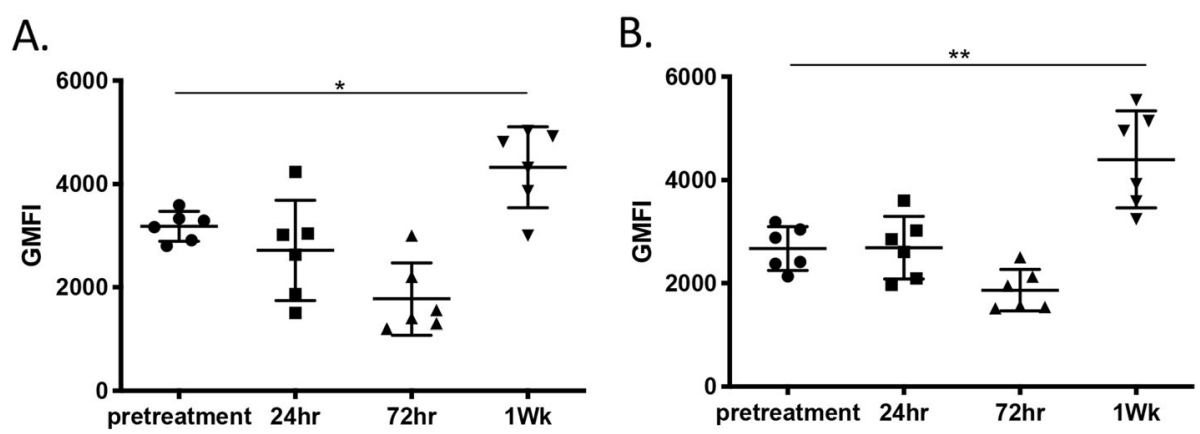

Fig. $6 \mathrm{MHCll}$ expression by monocytes in nose and oropharynx to LTC treatment. Nasal lavage and oropharyngeal swab samples were collected from dogs $(n=6)$ following treatment with LTC, and immunostained for analysis by flow cytometry, as noted in Methods. Monocytes from nasal lavage (a) and oropharyngeal (b) specimens were analyzed for expression of MHCll, prior to treatment and again at $24 \mathrm{~h}, 72 \mathrm{~h}$, and $7 \mathrm{~d}$ following LTC administration. Data were presented as geometric mean fluorescent intensity (gMFI) and analyzed using one-way ANOVA with multiple means comparisons. $\left.{ }^{*}, P \leq 0.05,{ }^{*}, P \leq 0.01\right)$

\section{Non-specific induction of anti-herpesvirus immunity by LTC treatment}

The preceding studies indicated that mucosal delivery of LTC to healthy dogs triggered local innate immune activation. Prior studies in rodent and cat models also reported that LTC treatment administered intranasally elicited strong antiviral activity [22-24]. Therefore, we used the opportunity provided by an inadvertent canine herpesvirus outbreak in 21 research Beagle puppies to evaluate the potential effectiveness of LTC as an early immunotherapy (see the Materials and Methods). Nucleic acids from canine herpesvirus, but not from the
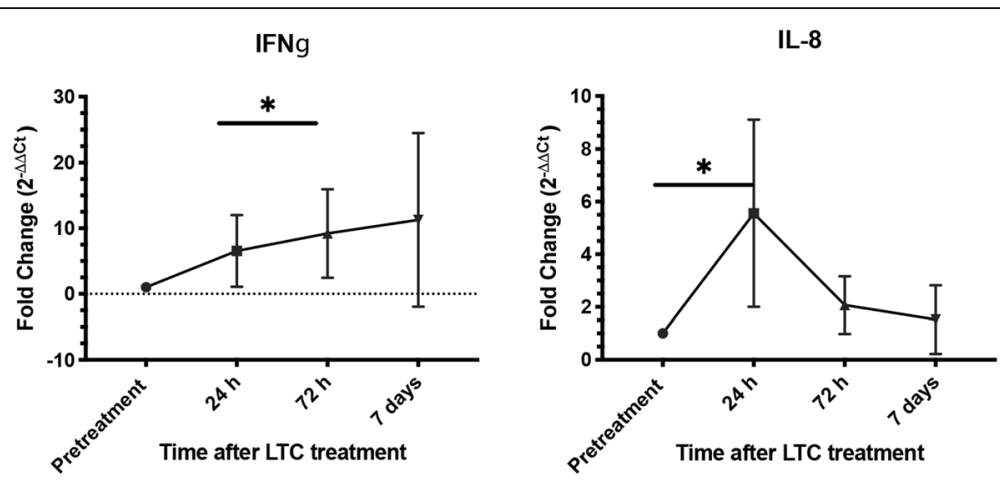

IL12p40

MCP-1
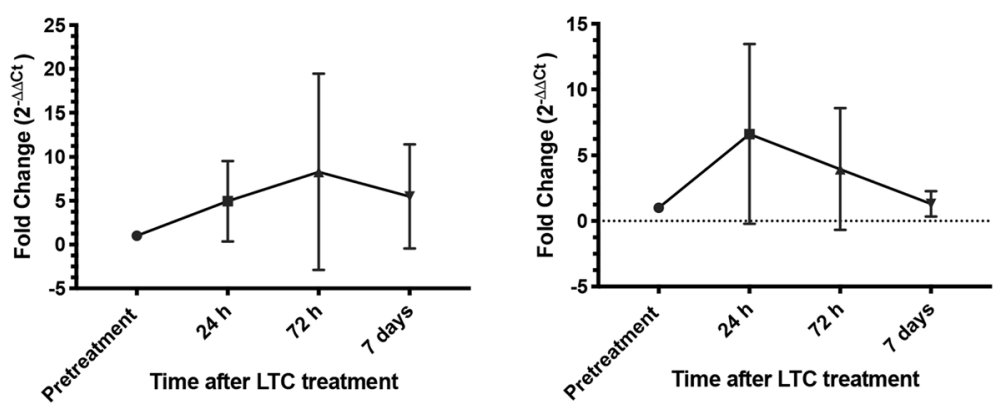

Fig. 7 Induction of inflammatory cytokine gene expression in oropharynx of dogs following mucosal administration of LTC. Samples were obtained from oropharyngeal swabs of dogs $(n=6)$ before and after treatment with LTC. At the indicated time points, RNA was extracted from swab samples and expression of 4 key inflammatory cytokine genes (IFNy, IL-8, IL-12, and MCP-1) was assessed using gRT-PCR, as noted in Methods. Data were expressed as fold-change in mRNA transcript levels over time. Significance was determined using the Wilcoxon signed-rank test, with ${ }^{*}, P \leq 0.05$ 
other canine viruses that were screened, were amplified from 20 of the 21 puppies. Based on this finding, a diagnosis of canine herpesvirus infection was made. Conjunctivitis was the most consistent clinical finding in affected dogs. The puppies were housed in 3 rooms, each containing clinically ill and PCR positive puppies. The LTC treatment was administered once to each of 7 puppies $(0.5 \mathrm{ml}$ LTC per nostril and $2 \mathrm{ml}$ orally), while 14 puppies were maintained as untreated controls. The proportions of observation days ( 40 days in total) in which treated animals (13.3\% of total observation days) and control, untreated dogs (35\% of total observation days) had evidence of conjunctivitis were calculated and compared (Fig. 8). The difference in conjunctivitis positive observation days was statistically significant when LTC-treated and control dogs were compared and suggested that a single administration of LTC induced a therapeutic response consistent with induction of early anti-viral immunity.

\section{Impact of LTC treatment on the oropharyngeal microbiome}

The microbiome of the upper respiratory tract and GI tract is known to be relatively stable over time in healthy individuals but can be perturbed significantly by administration of antimicrobial drugs [27-29]. However, much less is known about the impact of local activation of innate immune responses on the composition of the microbiome [30, 31]. For example, it is possible that

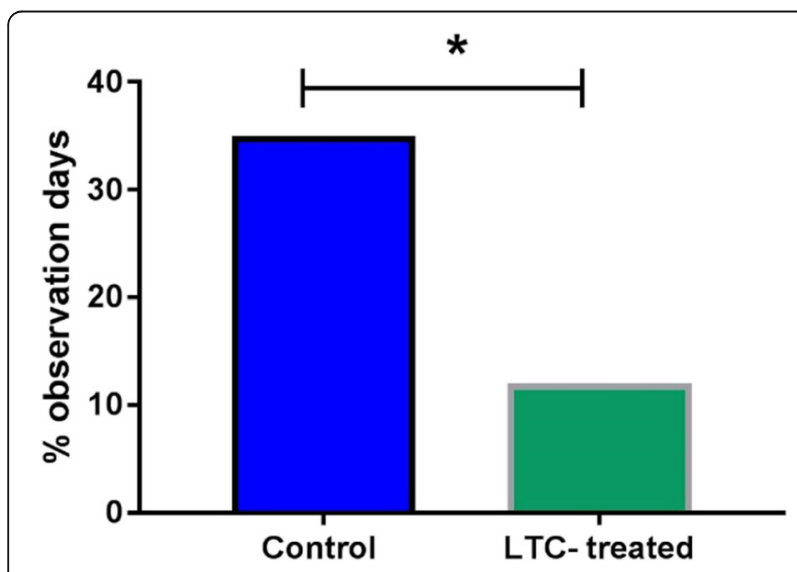

Fig. 8 Non-specific induction of antiviral immunity in dogs by LTC treatment. Purpose bred beagles $(n=21)$ shortly after exposure to canine herpesvirus were randomly assigned to treatment groups, with $n=7$ receiving a single intranasal and oral LTC treatment, and $n=14$ being treated with PBS, as noted in Methods. Dogs were monitored for clinical signs of herpesvirus infection, including conjunctivitis scores, as noted in Methods. The overall mean conjunctivitis score for each group was calculated and plotted. Statistical significance was evaluated by Fisher's exact test $\left(^{*}=p<\right.$ 0.05). The majority of clinical signs were recorded in the first 7 days after initiation of treatment (94\%) and none of the dogs had clinical signs consistent with canine herpesvirus after Day 13 local activation of immune defenses, including cytokines derived from epithelial cells and immune cells, could significantly alter the composition of the bacterial flora by either depleting or expanding certain bacterial populations. Alternatively, it is possible that the local microbiome may be relatively resistant to such immune perturbations. To address this question, the overall composition of the microbiome of the oropharynx in dogs $(n=6)$ that were treated with LTC was determined by 16S RNA sequencing immediately prior to treatment and again at 7 days and 14 days after treatment. Oropharyngeal samples were obtained by swabs and extracted microbial DNA was sequenced using an Illumina platform (Novogen, San Diego, CA). Data were analyzed as described in Methods.

Based on the results of 16S RNA sequencing (Fig. 9), it was apparent that the major phyla of bacteria located in the oropharynx of dogs did not change in any meaningful way following mucosal delivery of LTC. For example, significant changes in the relative abundance of major bacterial phyla including Spirochaetes, Fusobacteria, Actinobacteria, Firmicutes, Bacteroidetes, and Fusobacteria (except on day 14 for Fusobacteria) were not observed when pre-treatment abundances were compared to day 7 or day 14 post treatment abundances. Thus, while LTC administration transiently activated innate immune responses within the oropharyngeal region of dogs, the microbiome of the oropharynx appeared to be relatively resistant to perturbation by immune stimuli. These findings suggest that the local microbiome in healthy animals is relatively resistant to perturbation by the local effects of innate immune activation.

\section{Discussion}

Despite advances in vaccine technologies and availability, there remains an unmet need for new non-specific immunotherapeutics for dogs for prophylaxis or early therapy of infectious diseases. To address this need, we describe here a new mucosally active immunotherapy that has the potential to be used to prevent or treat early viral or bacterial infections or to treat established infections where strong induction of type I innate immune responses may lead to pathogen clearance. Moreover, there is also evidence that strong activation of innate immune responses and IFNY production can synergize with conventional antibiotics to clear chronic, intracellular bacterial pathogens. For example, we demonstrated previously that activation of innate immunity with LTC in rodent models of Burkholderia and Francisella infection significantly augmented the effectiveness of antibiotic therapy $[20,21]$.

Our in vitro studies with LTC revealed strong activation of production of key innate immune cytokines, including IL-8, MCP-1, IL-12p40, IFN $\gamma$ and TNF $\alpha$ 
A.

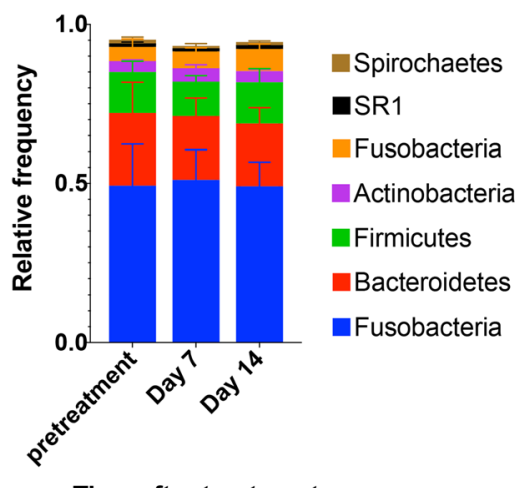

B.

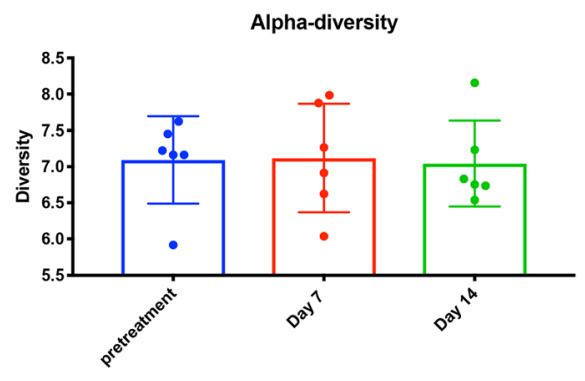

Time after treatment

Time after treatment

Fig. 9 Impact of LTC administration on oropharyngeal microbiome of dogs. Healthy dogs $(n=6)$ were treated by intranasal and oral administration of LTC, and oropharyngeal swabs were collected for evaluation of the resident microbiome, using 165 sequencing, as described in Methods. At the treatment times indicated, the relative abundances of key major phyla (Spirochaetes, Fusobacteria, Actinobacteria, Firmicutes, Bacteroidetes, and Fusobacteria) were determined by 165 sequencing and represented in panel (a). In panel (b), the alpha-diversity of all 6 phyla were compared over a 2-week period (b). These analyses did not detect significant differences in the phyla composition, or in the alpha diversity, as assessed by ANOVA

following LTC treatment in dog PBMC cultures and oropharyngeal cells (see Figs. 1, 2 and 7). We also found that LTC were taken up and internalized efficiently by canine epithelial cells following in vitro incubation (Fig. 4). The ability to adhere to and enter epithelial cells is important, because sustained contact with epithelial cells and immune cells in the nasal cavity and oropharynx is important for LTC to be internalized, engage TLR3 and TLR9 receptors in the endosomal compartment, and to activate sustained innate immune responses. Importantly, we also demonstrated that application of LTC to mucosal surfaces of the upper airways and oropharynx of dogs elicited local immune activation, as reflected by an increase in leukocyte recruitment and activation of cytokine production (see Figs. 5 and 6).

Infiltration of monocytes and lymphocytes into both the nasal cavity and the oropharynx provided direct evidence of LTC stimulation of local innate immune activation and chemokine production. Activation of cytokine production was also confirmed by RT-PCR analysis (see Fig. 7). In both the nose and the oropharynx of dogs, the cellular infiltration generally peaked at $72 \mathrm{~h}$ after LTC administration. Overall cellularity remained elevated in the nose for at least 7 days (the last time point evaluated), whereas cell counts returned to normal in the oropharynx by 7 days. Leukocyte infiltration was much more robust in the nose, and consisted of strong increases in $\mathrm{T}$ cells, monocytes, and $\mathrm{B}$ cells, with relatively smaller increases in neutrophils. In contrast, cellular infiltration in the oropharynx was dominated by myeloid cells (monocytes, neutrophils) with smaller $\mathrm{T}$ and $\mathrm{B}$ cell responses. These differences most likely reflect local differences in chemokine production in response to TLR stimulation, as well as heterogeneity in resident tissue cell populations. Overall, the enhanced leukocyte infiltration into upper airway tissues following LTC administration could enhance both antiviral and antibacterial immune defenses, via direct mechanisms of control (e.g., macrophage bactericidal activity, see Fig. 3) or via indirect mechanisms such as cytokine and antibody production.

We also demonstrated that LTC treatment induced therapeutic anti-viral immunity in dogs when administered early after canine herpesvirus infection. For example, in dogs infected in a canine herpesvirus outbreak, we found that early mucosal administration of LTC generated significant reduction in clinical signs (e.g., conjunctivitis) associated with canine herpesvirus infection (see Fig. 8). Similar results were also observed recently in cats treated with LTC shortly prior to intranasal challenge with FHV-1, where a significant reduction in clinical signs of infection and viral shedding was observed $[22,24]$. We have also observed complete or nearly complete anti-viral and anti-bacterial protection in a number of different lethal rodent models of infection following intranasal administration of an earlier version of LTC $[20,21,23]$. Thus, it is apparent that LTC mucosal immunotherapy can elicit significant antiviral immunity in at least 3 different animal species (mouse, dog, cat).

\section{Conclusion}

In summary, we have demonstrated using in vitro and in vivo studies that a new immunotherapeutic consisting of liposomes complexed to TLR3 and TLR9 agonists 
potently activated innate immune responses in dogs. The immune responses triggered by intranasal and oral administration were primarily concentrated locally, based on evidence of cellular immune infiltrates at the sites of administration and the lack of systemic responses (e.g., fever) to treatment. These LTC immune complexes have potential utility as a new method of rapidly eliciting non-specific protective immunity in the event of an outbreak (e.g., canine influenza virus or Bordetella infections in boarding facilities) while potentially also having a direct translational utility against infections in humans and other species. Moreover, concurrent administration of LTC with conventional vaccines may improve or accelerate overall vaccine immunity. Finally, increasingly veterinarians will be encouraged to seek nonantimicrobial alternatives to disease prevention or therapy, and the use of potent, mucosally active immunotherapeutics such as LTC are likely to have an important role to play in these new approaches.

\section{Methods}

Preparation of liposome-TLR complexes (LTC)

Liposomes were prepared by drying cationic lipid 1,2dioleoyl-3-trimentylammonium-propane (DOTAP) and cholesterol (Avanti Polar Lipids, Alabaster, AL) to a thin film on glass tubes, using a vacuum desiccator. The lipids were then rehydrated to form liposomes, as described previously [16, 24, 32]. Polyinosinic, polycytidylic acid (pIC; InVivoGen, San Diego, CA) and non-coding commercial plasmid DNA (PCR2.1, Life Sciences, CA) were added to the liposomes to form liposome-TLR agonist complexes (LTC), as reported previously [16, 24, 32]. The final concentration of both TLR agonists were $25 \mu \mathrm{g} / \mathrm{ml}$. The endotoxin content of the plasmid was < $0.25 \mathrm{EU} / \mathrm{ug}$ and pIC was certified to be LPS-free. For in vitro tracking studies, liposomes were formulated to contain 10\% (v/v) TopFluor-labeled cholesterol (Avanti, Alabaster, AL).

\section{In vitro cell activation with LTC}

To assess cell activation, peripheral blood mononuclear cells (PBMC) from healthy dogs were prepared as described previously [33]. Briefly, whole blood was obtained by jugular venipuncture and collected into EDTA tubes, then diluted 1:2 with sterile PBS, layered over a Ficoll (GE Healthcare, Uppsala, Sweden) gradient and centrifuged for $30 \mathrm{~min}$. Following centrifugation, PBMC were collected from the Ficoll interface and washed twice in PBS and then re-suspended in complete tissue culture medium consisting of DMEM (Thermo Fisher Scientific, Watham, MA) medium containing $10 \%$ FBS (VWR Seradigm, Denver, CO), essential and non-essential amino acids, penicillin and streptomycin (Gibco-Thermo Fisher Scientific, Pittsburgh, PA).
After counting, cells were plated in 96-well flat bottom plates (Celltreat, Pepperell, MA) at a density of 1 $\mathrm{X} 10^{6}$ cells/well in $200 \mu \mathrm{l}$ medium. For PBMC activation, LTC were added at 4 different dilutions $(0.02 \mu \mathrm{l} /$ well, $0.2 \mu \mathrm{l}$ per well, $1 \mu \mathrm{l}$ per well, and $2.0 \mu \mathrm{l}$ per well) in triplicate wells of PBMC in $200 \mu \mathrm{l}$ complete DMEM, with careful mixing, and the cells were then incubated for an additional 24-48 h. Conditioned medium was collected for IFNY and TNF $\alpha$ assays and cells analyzed via flow cytometric analysis for modulation of activation markers (see below). PBMC were assayed at least twice using separate donor animals.

\section{Generation of monocyte-derived macrophages}

To generate monocyte-derived macrophages (MDM) in vitro, PBMC were plated in 24-well plates at a density of $5 \times 10^{6}$ cells $/ \mathrm{ml}$ and allowed to adhere for $4 \mathrm{~h}$, after which non-adherent cells were removed by gentle washing with PBS. The adherent cells were then cultured in complete DMEM with the addition of $10 \mathrm{ng} / \mathrm{ml}$ recombinant human M-CSF (R\&D systems,) for 7 days, and replacing with fresh M-CSF medium every 3 days. This technique produced nearly pure cultures of dog macrophages, as assessed by flow cytometry and immunostaining for CD11b expression (data not shown).

\section{Analysis of cytokine gene expression by qRT-PCR}

Samples were obtained from oropharyngeal swabs of dogs $(n=6)$ before and after treatment with LTC, and expression of IL-8, MCP-1, IL-12p40, and IFN $\gamma$ genes was determined via quantitative real time-(qRT)-PCR, using previously published primers [34, 35]. Briefly, cDNA was prepared by isolation of RNA followed by reverse transcription using a commercial kit (Qiagen, Germantown, MD) followed by amplification using $\mathrm{SYBR}^{\mathrm{Tw}}$ green primers (Bio-Rad, Hercules, CA). Amplification was performed using a qPCR MX3000p system instrument (Agilent, Santa Clara, CA). All primers were validated to have an efficiency $>90 \%$ using stimulated and unstimulated healthy dog PBMC. pRT-PCR was used to quantify cytokine transcript levels as shown previously [24].

\section{IFNY and TNFa ELISA}

Supernatants from PBMC cultures were analyzed for IFN $\gamma$ and TNF $\alpha$ using commercial canine IFN $\gamma$ (DuoSet ${ }^{\circ}$ Canine IFNY kit; R\&D systems, Minneapolis, MN) and TNF $\alpha$ (DuoSet ${ }^{\circ}$ Canine TNF $\alpha$ kit; R\&D systems, Minneapolis, MN) according to manufacturer's protocols.

Flow cytometry for analysis of in vitro activated cells and nasal and oropharyngeal lavage specimens

Cells cultured in vitro were harvested after $24 \mathrm{~h}$ of LTC stimulation and immunostained with fluochrome-conjugated antibodies: T cells: CD5-PE; CD4-PB, CD8-APC; B 
cells: MHCII-FITC, CD21-APC and CD45-PB; monocytes: MHCII-FITC, CD14-APC, CD11b-PC7, CD45-PB. Prior to adding staining antibody, cells were preincubated for $5 \mathrm{~min}$ with normal dog serum containing human IgG and anti-mouse FcRIII antibodies to block any non-specific antibody binding. Cells were subsequently immunostained with the conjugated antibodies for 20 min at $4{ }^{\circ} \mathrm{C}$ in FACs buffer (PBS with $2 \%$ FBS and $0.05 \%$ sodium azide).

Nasal and oropharyngeal samples obtained by gentle swabbing of the dogs (see below) were transferred to 50 $\mathrm{ml}$ conical tubes containing $25 \mathrm{ml}$ of sterile PBS and the sampling swabs were used to gently stir the solution to dislodge cellular material from the swabs. The solution was then filtered through $50 \mu \mathrm{m}$ cell strainers (Corning, Fairport, NY) and pelleted by centrifugation. Cells from the resuspended pellet were washed once with PBS then stained directly with conjugated antibodies described above. Additional aliquots of cells were stained with isotype-matched antibodies as controls for each primary antibody. Flow cytometric analysis was performed using a Beckman Coulter Gallios flow cytometer (Beckman Coulter, Indianapolis, IN), and data were analyzed using FlowJo software (Tree Star, Ashland, OR).

\section{Animal studies}

All animal studies were approved by the Institutional Animal Care and Use Committee (IACUC) at a contract research facility in Fort Collins, CO (Protocol \# 170024). Purpose-bred Beagle dogs used for these the studies were purchased from a commercial vendor. Dogs were housed in large kennels at the facility under standard light vs. dark conditions and cared for by qualified animal care technicians. Following completion of the studies, all dogs were adopted to local owners in the Ft. Collins area.

\section{Administration of liposome-TLR complexes to study animals}

A power calculation indicated that the minimum number of dogs to be used in this study was $n=6$ for each group. To minimize the number of research animals subjected to treatment, we chose to examine a total of 12 dogs: Group 1: 6 untreated and Group 2: 6 LTCtreated. Study dogs ( $n=6$ per group) were treated by a single administration of LTC by the intranasal and oral routes. For LTC-treated dogs, $0.5 \mathrm{ml}$ LTC was administered to each nostril, using a $1 \mathrm{ml}$ syringe and minimal manual restraint. In addition, each dog also received 2.0 $\mathrm{ml}$ of LTC administered orally, directed to the back of the throat, using a $3 \mathrm{ml}$ syringe. Control animals $(n=6)$ were administered sterile PBS, $0.5 \mathrm{ml}$ per nostril and 2.0 $\mathrm{ml}$ orally. The intranasal dose of each TLR ligand for each dog was approximately $20 \mu \mathrm{g}$ per $\mathrm{kg}$ body weight.
Collection of nasal lavage and oropharyngeal samples

To collect nasal lavage samples, dogs were briefly restrained manually in a head-down position, and $5 \mathrm{ml}$ of pre-warmed sterile PBS solution was quickly administered into each nostril, and the fluid backflow was collected from the nostrils in $15 \mathrm{ml}$ conical tubes. PBS was administered once more and pooled fluid from each dog was stored on ice. Oropharyngeal samples were collected by gently swabbing the caudal oropharyngeal region by gently rubbing and rolling against the mucosa. Swabs were placed in $15 \mathrm{ml}$ conical tubes containing complete DMEM tissue culture medium containing 10\% FBS and stored on ice. Both Nasal and oropharyngeal samples were collected $24 \mathrm{~h}$ prior to administration of LTC and samples were likewise obtained again at $72 \mathrm{~h}$ and 7 days post-LTC treatment.

\section{Preparation and analysis of nasal and oropharyngeal samples}

Nasal lavage fluid was diluted with $3 \mathrm{ml}$ of complete DMEM and oropharyngeal swabs were collected from the distal oropharynx and immediately placed in $2 \mathrm{ml}$ of complete tissue culture medium in $15 \mathrm{ml}$ polypropylene tubes on ice. To dislodge cells from the swabs, the samples were lightly vortexed. Fluid was subsequently transferred to a new tube and the swabs were again rinsed with $2 \mathrm{ml}$ of PBS and pooled with the first wash. Pooled samples were filtered through a $70 \mu \mathrm{m}$ cell strainer (CellTreat, Pepperell, MA) to remove large debris and mucus. Samples were then centrifuged and the pellets were resuspended in $0.5 \mathrm{ml}$ of PBS and $10 \mu \mathrm{l}$ aliquots were stained with $0.4 \%$ trypan blue and blue-excluding cells were counted using a Nexcelom Cellometer ${ }^{\text {ma }}$ Auto T4 cell counter (Nexcelom; Lawrence, MA). Nasal lavage samples were processed similarly. To determine cell types and activation states, equivalent numbers of cells were processed and stained for flow cytometric analyses as described above.

\section{Non-specific induction of anti-herpesvirus immunity by LTC treatment}

Beagle puppies $(n=21)$ that were originally purchased for a separate study but developed sneezing and conjunctivitis had orophargygeal swabs collected as described. The samples were assessed by a PCR panel performed at a commercial laboratory (Antech Diagnostics, Lake Success, NY) and the only viral nucleic acids of common canine pathogens that were amplified were those of canine herpesvirus (detected in 20 of 21 animals). The affected 21 dogs were randomly allocated to housing in 3 different rooms of 7 dogs each. Each room contained clinically ill and canine herpesvirus PCR positive dogs. Within $24 \mathrm{~h}$ of the first recognized clinical signs, 7 dogs were administered $0.5 \mathrm{ml}$ LTC per nostril 
and $2 \mathrm{ml}$ orally, while 14 dogs were maintained as untreated controls. Two trained and study-blinded observers then applied a standardized clinical score rubric to dogs in each room for $30 \mathrm{~min}$ each day for 40 days. The primary clinical sign noted over the course of the study was conjunctivitis. The proportions of 40 total observation days in which treated and control dogs had evidence of conjunctivitis were calculated and compared by means of Fisher's exact test (Fig. 8).

\section{$16 \mathrm{~S}$ sequencing of bacterial microbiomes in oropharyngeal samples}

Oropharyngeal swabs from dogs were collected prior to LTC treatment, in addition $24 \mathrm{~h}, 72 \mathrm{~h}$ and 7 days post treatment. Bacterial pellets collected from swabs were concentrated by high speed centrifugation stored in PBS at $-20^{\circ} \mathrm{C}$ until processing for DNA extraction. Microbial DNA extraction was performed using a MoBio Powersoil DNA Isolation kit (Qiagen, Valencia, CA) according to manufacturer's protocol. 16S rRNA sequencing was performed by Novogene (Chula Vista, CA). Negative controls were verified on Nanodrop 1000 to have $<2 \mathrm{ng} / \mathrm{uL}$ of total DNA. DNA concentration and purity was monitored on $1 \%$ agarose gels.

The ribosomal RNA genes of the bacterial V4 region were amplified using V4: 515F-806R in accordance with the Earth Microbiome project [36]. All amplification reactions were carried out in Phusion High-Fidelity PCR Master Mix (New England Biolabs, MA). PCR products, sequencing libraries library quality and sequencing were generated, assessed and performed respectively as described in [37]. Sequence quality control, adapter trimming and feature table construction were performed according to the QIIME2 version 2018.2 demux summarize DADA2 [38].

Operational Taxonomic Units (OTUs) were resolved at $97 \%$ sequence similarity using QIIME as described in $[37,39]$. For taxonomic assignment, the Greengenes $16 \mathrm{~S}$ database was used at 0.8 confidence level. Phylogenetic tree was constructed using Qiime2 phylogeny fasttree [40]. Alpha diversity and beta diversity (weighted and unweighted unifractions) was calculated using Qiime2 diversity core metrics [41]. Differential abundance testing was performed using Analysis of Composition of Microbiomes (ANCOM) [42]. Significance in relative abundance on Phylum, Class, Order, Family and Genus levels was calculated using 2-way ANOVA with Tukey post-test. Graphical results were plotted using Graph Pad Prism 8 (GraphPad Software, La Jolla California USA).

\section{Confocal microscopy and cell imaging}

To assess uptake of LTC by relevant target cells in the nasal cavity and oropharynx (ie, squamous epithelium and macrophages), LTC were incubated with canine squamous cell carcinoma cells (cell line CSCAG891, generated in the Dow lab) and with canine macrophages (not shown). Cells were incubated with serial dilutions of LTC prepared with a fluorescent liposome (TopFluor, Avanti Polar Lipids, Alabaster, AL) for tracking. After the indicated periods of incubation, the cells were fixed and then imaged using an Olympus (Waltham, MA) IX3 confocal microscope. Images were processed and analyzed using Olympus CellSens ${ }^{\circ}$ software.

\section{Macrophage killing assays}

Monocyte-derived macrophages (MDM) were derived as described above and were plated at a density of $1 \times 10^{5}$ cells per well in 48-well plates and subsequently either left untreated or treated with LTC at $5 \mu \mathrm{l} / \mathrm{ml}$ or $10 \mathrm{ng} /$ $\mathrm{ml}$ canine IFN $\gamma$ (data not shown in Fig. 3) in $500 \mu \mathrm{l}$ for $24 \mathrm{~h}$. Untreated or treated MDM were infected at MOI $=5$ in $\mathrm{HBSS}$ containing $\mathrm{Ca}^{++}$and $\mathrm{Mg}^{++}$in $10 \%$ dog serum for $1 \mathrm{hr}$. To enumerate intracellular concentrations of bacteria, MDM were lysed with sterile distilled water and diluted 10-fold serially in PBS and plated on quadrants of brain heart infusion (BHI) plates. Some cultures were stopped immediately after infection to determine initial intracellular bacterial concentrations, while others were incubated for an additional $2 \mathrm{~h}$ to allow for bacterial killing prior to enumeration of remaining intracellular bacterial concentrations. Bacterial colony forming units (CFU) were determined and CFU from the $1 \mathrm{~h}$ incubation was compared to the $2 \mathrm{~h}$ incubation to determine intracellular killing efficiency. The remaining CFU after $2 \mathrm{~h}$ was divided by the CFU from the $1 \mathrm{~h}$ incubation and this quotient was multiplied by 100 represented the percent killing of MRSP by the treated or untreated MDM.

\section{Statistical methods}

For comparisons between data sets with two treatment groups, statistical significance was evaluated by Fisher's exact test $(*=p \leq 0.05)$. ANOVA followed by Tukey multiple means post-test was used to perform analyses comparing 3 or more groups.

Statistical significance defined as $p \leq 0.05\left({ }^{*}\right)$. Analyses were done using Prism 8 software (GraphPad, La Jolla, CA).

\section{Abbreviations}

LTC: Liposome TLR complexes; MDM: Monocyte-derived macrophages; MHCII: Major histocompatibility complex class II; MRSP: Methicillin-resistant Staphlococcus pseudintermedius

\section{Acknowledgements}

The authors wish to thank students in the Center for Companion Animal Studies for their help with animal studies. 


\section{Authors' contributions}

Design of study and experiments: SD, ML and WW. Laboratory activities WW, LC, AK, SS and JK. Analysis of results and data interpretation: WW, SD and LC. Manuscript drafting: WW and SD. All authors read and approved the final manuscript.

\section{Funding}

These studies were supported by a grant from the State of Colorado Office of Economic Development and International Translation (OEDIT) and by a grant from the Center for Companion Animal Studies at Colorado State University (CCASCSU). OEDIT is intended to support Colorado's bioscience industry and funds were used herein to fund materials and salary support for LTC sample collection, research supplies, data analysis and publication costs. CCASCSU funds were used as salary support for those involved in treatment of dogs with LTC and collection of biological samples.

\section{Availability of data and materials}

All data generated and/or analyzed during this study are included in this published article. Microbiome data has been uploaded to the European Nucleotide Archive (ENA) (primary accession \# PRJEB34170) https:// www.ebi.ac.uk/ena/submit/sra/\#studies.

\section{Ethics approval and consent to participate}

All animal studies were approved by the Institutional Animal Care and Use Committee (IACUC) at a contract research facility in Fort Collins, CO (Protocol \# 170 024). Purpose-bred Beagle dogs used for these the studies were purchased from a commercial vendor. Following completion of the studies, all dogs were adopted to local owners in the Ft. Collins area.

\section{Consent for publication}

Not applicable.

\section{Competing interests}

ML and SD both hold stock options and corporate positions in Poudre Canyon Therapeutics, a Fort Collins company developing the LTC immunotherapy platform technology.

Received: 8 July 2019 Accepted: 3 September 2019

Published online: 13 September 2019

\section{References}

1. Priestnall SL, Mitchell JA, Walker CA, Erles K, Brownlie J. New and emerging pathogens in canine infectious respiratory disease. Vet Pathol. 2014;51(2): 492-504.

2. Lavan $R$, Knesl O. Prevalence of canine infectious respiratory pathogens in asymptomatic dogs presented at US animal shelters. J Small Anim Pract. 2015;56(9):572-6

3. Lappin MR, Blondeau J, Boothe D, Breitschwerdt EB, Guardabassi L, Lloyd DH, Papich MG, Rankin SC, Sykes JE, Turnidge J, et al. Antimicrobial use guidelines for treatment of respiratory tract disease in dogs and cats: antimicrobial guidelines working Group of the International Society for companion animal infectious diseases. J Vet Intern Med. 2017:31(2):279-94.

4. Hoelzer K, Murcia PR, Baillie GJ, Wood JL, Metzger SM, Osterrieder N, Dubovi EJ, Holmes EC, Parrish CR. Intrahost evolutionary dynamics of canine influenza virus in naive and partially immune dogs. J Virol. 2010;84(10): 5329-35.

5. Alexandroff AB, Jackson AM, O'Donnell MA, James K. BCG immunotherapy of bladder cancer: 20 years on. Lancet. 1999;353(9165):1689-94.

6. van der Meijden AP. Non-specific immunotherapy with bacille CalmetteGuerin (BCG). Clin Exp Immunol. 2001;123(2):179-80.

7. MacEwen EG, Kurzman ID, Rosenthal RC, Smith BW, Manley PA, Roush JK, Howard PE. Therapy for osteosarcoma in dogs with intravenous injection of liposome-encapsulated muramyl tripeptide. J Natl Cancer Inst. 1989;81(12):935-8.

8. Kurzman ID, MacEwen EG, Rosenthal RC, Fox LE, Keller ET, Helfand SC, Vail DM, Dubielzig RR, Madewell BR, Rodriguez CO Jr, et al. Adjuvant therapy for osteosarcoma in dogs: results of randomized clinical trials using combined liposome-encapsulated muramyl tripeptide and cisplatin. Clin Cancer Res. 1995;1(12):1595-601.

9. Vail DM, MacEwen EG, Kurzman ID, Dubielzig RR, Helfand SC, Kisseberth WC, London CA, Obradovich JE, Madewell BR, Rodriguez CO Jr, et al. Liposome- encapsulated muramyl tripeptide phosphatidylethanolamine adjuvant immunotherapy for splenic hemangiosarcoma in the dog: a randomized multi-institutional clinical trial. Clin Cancer Res. 1995;1(10):1165-70.

10. MacEwen EG, Kurzman ID, Vail DM, Dubielzig RR, Everlith K, Madewell BR, Rodriguez CO Jr, Phillips B, Zwahlen CH, Obradovich J, et al. Adjuvant therapy for melanoma in dogs: results of randomized clinical trials using surgery, liposome-encapsulated muramyl tripeptide, and granulocyte macrophage colony-stimulating factor. Clin Cancer Res. 1999:5(12):4249-58.

11. Teske E, Rutteman GR, vd Ingh TS, van Noort R, Misdorp W. Liposomeencapsulated muramyl tripeptide phosphatidylethanolamine (L-MTP-PE): a randomized clinical trial in dogs with mammary carcinoma. Anticancer Res. 1998:18(2A):1015-9.

12. Nardin A, Lefebvre ML, Labroquere K, Faure O, Abastado JP. Liposomal muramyl tripeptide phosphatidylethanolamine: targeting and activating macrophages for adjuvant treatment of osteosarcoma. Curr Cancer Drug Targets. 2006;6(2):123-33.

13. Kleinerman ES, Jia SF, Griffin J, Seibel NL, Benjamin RS, Jaffe N. Phase ॥ study of liposomal muramyl tripeptide in osteosarcoma: the cytokine cascade and monocyte activation following administration. J Clin Oncol. 1992;10(8):1310-6.

14. Guth A, Dow S. Withrow and MacEwen's small animal clinical oncology; 2013.

15. Kurzman ID, Shi F, Vail DM, MacEwen EG. In vitro and in vivo enhancement of canine pulmonary alveolar macrophage cytotoxic activity against canine osteosarcoma cells. Cancer Biother Radiopharm. 1999;14(2):121-8.

16. Dow S. Liposome-nucleic acid immunotherapeutics. Expert Opin Drug Deliv. 2008:5(1):11-24.

17. Kamstock D, Guth A, Elmslie R, Kurzman I, Liggitt D, Coro L, Fairman J, Dow S. Liposome-DNA complexes infused intravenously inhibit tumor angiogenesis and elicit antitumor activity in dogs with soft tissue sarcoma. Cancer Gene Ther. 2006;13(3):306-17.

18. Henderson A, Propst K, Kedl R, Dow S. Mucosal immunization with liposome-nucleic acid adjuvants generates effective humoral and cellular immunity. Vaccine. 2011;29(32):5304-12.

19. Jones A, Bosio C, Duffy A, Goodyear A, Schriefer M, Dow S. Protection against pneumonic plague following oral immunization with a nonreplicating vaccine. Vaccine. 2010;28(36):5924-9.

20. Troyer RM, Propst KL, Fairman J, Bosio CM, Dow SW. Mucosal immunotherapy for protection from pneumonic infection with Francisella tularensis. Vaccine. 2009;27(33):4424-33.

21. Goodyear A, Kellihan L, Bielefeldt-Ohmann H, Troyer R, Propst K, Dow S. Protection from pneumonic infection with burkholderia species by inhalational immunotherapy. Infect Immun. 2009;77(4):1579-88.

22. Contreras ET, Olea-Popelka F, Wheat W, Dow S, Hawley J, Lappin MR. Evaluation of liposome toll-like receptor ligand complexes for non-specific mucosal immunoprotection from feline herpesvirus-1 infection. J Vet Intern Med. 2019:33(2):831-7.

23. Logue CH, Phillips AT, Mossel EC, Ledermann JP, Welte T, Dow SW, Olson KE, Powers AM. Treatment with cationic liposome-DNA complexes (CLDCs) protects mice from lethal Western equine encephalitis virus (WEEV) challenge. Antivir Res. 2010;87(2):195-203.

24. Wheat W, Chow L, Coy J, Contreras E, Lappin M, Dow S. Activation of upper respiratory tract mucosal innate immune responses in cats by liposomal toll-like receptor ligand complexes delivered topically. J Vet Intern Med. 2019;33(2):838-45.

25. Shah V, Taratula O, Garbuzenko OB, Patil ML, Savla R, Zhang M, Minko T. Genotoxicity of different nanocarriers: possible modifications for the delivery of nucleic acids. Curr Drug Discov Technol. 2013;10(1):8-15.

26. Italiani $\mathrm{P}$, Boraschi D. From monocytes to M1/M2 macrophages: phenotypical vs. Functional Differentiation. Front Immunol. 2014:5:514

27. Yoon MY, Yoon SS. Disruption of the gut ecosystem by antibiotics. Yonsei Med J. 2018;59(1):4-12.

28. Le Noci V, Guglielmetti S, Arioli S, Camisaschi C, Bianchi F, Sommariva M, Storti C, Triulzi T, Castelli C, Balsari A, et al. Modulation of pulmonary microbiota by antibiotic or probiotic aerosol therapy: a strategy to promote Immunosurveillance against lung metastases. Cell Rep. 2018; 24(13):3528-38

29. Becattini S, Taur Y, Pamer EG. Antibiotic-induced changes in the intestinal microbiota and disease. Trends Mol Med. 2016;22(6):458-78.

30. Shi N, Li N, Duan $X$, Niu H. Interaction between the gut microbiome and mucosal immune system. Mil Med Res. 2017;4:14. 
31. Koch BEV, Yang S, Lamers G, Stougaard J, Spaink HP. Intestinal microbiome adjusts the innate immune setpoint during colonization through negative regulation of MyD88. Nat Commun. 2018;9(1):4099.

32. Zaks K, Jordan M, Guth A, Sellins K, Kedl R, Izzo A, Bosio C, Dow S. Efficient immunization and cross-priming by vaccine adjuvants containing TLR3 or TLR9 agonists complexed to cationic liposomes. J Immunol. 2006;176(12): 7335-45.

33. Wheat WH, Chow L, Kurihara JN, Regan DP, Coy JW, Webb TL, Dow SW. Suppression of canine dendritic cell activation/maturation and inflammatory cytokine release by mesenchymal stem cells occurs through multiple distinct biochemical pathways. Stem Cells Dev. 2017;26(4):249-62.

34. Tamura Y, Ohta H, Yokoyama N, Lim SY, Osuga T, Morishita K, Nakamura K, Yamasaki M, Takiguchi M. Evaluation of selected cytokine gene expression in colonic mucosa from dogs with idiopathic lymphocytic-plasmacytic colitis. J Vet Med Sci. 2014:76(10):1407-10

35. Vitoriano-Souza J, Moreira N, Menezes-Souza D, Roatt BM, de Oliveira Aguiar-Soares RD, Siqueira-Mathias FA, de Oliveira Cardoso JM, Giunchetti $R C$, de Sa RG, Correa-Oliveira R, et al. Dogs immunized with LBSap vaccine displayed high levels of IL-12 and IL-10 cytokines and CCL4, CCL5 and CXCL8 chemokines in the dermis. Mol Immunol. 2013;56(4):540-8.

36. Gohl DM, Vangay P, Garbe J, MacLean A, Hauge A, Becker A, Gould TJ, Clayton JB, Johnson TJ, Hunter R, et al. Systematic improvement of amplicon marker gene methods for increased accuracy in microbiome studies. Nat Biotechnol. 2016;34(9):942-9.

37. Soontararak S, Chow L, Johnson V, Coy J, Wheat W, Regan D, Dow S. Mesenchymal stem cells (MSC) derived from induced pluripotent stem cells (iPSC) equivalent to adipose-derived MSC in promoting intestinal healing and microbiome normalization in mouse inflammatory bowel disease model. Stem Cells Transl Med. 2018;7(6):456-67.

38. Caporaso JG, Kuczynski J, Stombaugh J, Bittinger K, Bushman FD, Costello EK, Fierer N, Pena AG, Goodrich JK, Gordon Jl, et al. QIIME allows analysis of high-throughput community sequencing data. Nat Methods. 2010;7(5):335-6.

39. Waterman KC, Gerst P, Macdonald BC. Relative humidity hysteresis in solidstate chemical reactivity: a pharmaceutical case study. J Pharm Sci. 2012; 101(2):610-5.

40. Price MN, Dehal PS, Arkin AP. FastTree 2--approximately maximumlikelihood trees for large alignments. PLoS One. 2010;5(3):e9490.

41. Vazquez-Baeza Y, Pirrung M, Gonzalez A, Knight R. EMPeror: a tool for visualizing high-throughput microbial community data. Gigascience. 2013; 2(1):16.

42. Mandal S, Van Treuren W, White RA, Eggesbo M, Knight R, Peddada SD. Analysis of composition of microbiomes: a novel method for studying microbial composition. Microb Ecol Health Dis. 2015;26:27663.

\section{Publisher's Note}

Springer Nature remains neutral with regard to jurisdictional claims in published maps and institutional affiliations.

Ready to submit your research? Choose BMC and benefit from:

- fast, convenient online submission

- thorough peer review by experienced researchers in your field

- rapid publication on acceptance

- support for research data, including large and complex data types

- gold Open Access which fosters wider collaboration and increased citations

- maximum visibility for your research: over $100 \mathrm{M}$ website views per year

At $\mathrm{BMC}$, research is always in progress.

Learn more biomedcentral.com/submissions 